\title{
Are younger people sleepier than older people after missing bedtime and night sleep? It depends...
}

\author{
Arcady Putilov \\ Research Group for Math-Modeling of Biomedical Systems \\ Research Institute for Molecular Biology and Biophysics of the \\ Federal Research Centre for Fundamental and Translational \\ Medicine \\ Novosibirsk, Russia \\ 0000-0003-2779-9046
}

\begin{abstract}
Feeling sleepy might evolve for motivating humans and apes to initiate sleep-preparatory behaviors prior to their habitual bedtime. Sleep deprivation experiments consistently revealed that younger rather than older people are more vulnerable to sleep loss. If so, why don't they go to sleep earlier, become sleepier during the day and nap more frequently than older people? To address such questions, we obtained subjective and objective sleepiness measurements during prolongation of wakefulness of 130 and 48 volunteers to the next morning and to the next two days after today, respectively (ages ranged from 15 to 67 years). The results suggested that the answer to a question of whether sleep loss makes younger people sleepier than older people depends upon the method of sleepiness measurement, either objective (e.g., alpha rhythm attenuation in eyes closed condition) or subjective (e.g., alertness-sleepiness self-scoring). We concluded that the motivational function of sleepiness remains preserved in older adults despite a lowered responsiveness of their electroencephalogram to extension of wakefulness beyond its normal duration.
\end{abstract}

Keywords - EEG, alpha attenuation, aging, sleep loss, drowsiness, alertness

\section{INTRODUCTION}

Human aging is associated with notable changes in sleepwake behaviors. Older adults are often rising early, feeling sleepy during the day, taking a nap, going to bed early, having difficulty falling asleep, and suffering from frequent night sleep interruptions and early morning awakenings. However, studies of age-associated differences in response to night sleep deprivation consistently revealed that older adults are more resistant to impairment of alertness and performance than younger adults (reviewed with suggesting an explanation in $[1,2])$. Subjective sensation of sleepiness prior to habitual bedtime might evolve for motivating humans and apes to switch to sleep-preparatory behaviors from any other current daytime activities [3]. If younger adults become sleepier than older adults during prolongation of wakefulness, why don't they go to sleep earlier, become sleepier during the day and nap more frequently?

We addressed such questions by extending a period of wakefulness of people aged between 15 and 67 years to examine the effect of such an extension on subjective and objective measures of their sleepiness. The following two hypotheses were tested:

- An analysis of objective measure of sleepiness would provide further evidence for a reduced vulnerability of older adults to sleep deprivation;

- in contrast, an analysis of subjective measure of sleepiness would reveal age independence of the strength of feeling sleepy due to sleep loss.

\author{
Olga Donskaya \\ Research Group for Math-Modeling of Biomedical Systems \\ Research Institute for Molecular Biology and Biophysics of the \\ Federal Research Centre for Fundamental and Translational \\ Medicine \\ Novosibirsk, Russia \\ dolly@niimbb.ru
}

\section{METHODS}

\section{Stady Participants and Study Protocols}

The main electroencephalographic (EEG) dataset was collected in two-day sleep deprivation experiments with 48 study participants ( 27 females) aged between 15 and 67 years (mean age \pm standard deviation were $36.6 \pm 13.1$ years). The participants were grouped into three ages of 15-18 participants each (younger, 15-26, intermediate, 30-40, and older, 46-67 years). They arrived in the research unit on Friday before 18:30 to leave it on Sunday at 19:30 with an expectation to complete 25 resting EEG recording sessions separated by $2-\mathrm{h}$ intervals (2 and $5 \mathrm{~min}$ with eyes open and closed, respectively).

The additional EEG dataset was collected in a shorter (one-night) sleep deprivation experiments with 130 study participants (76 females) 15-67 year old (mean age \pm standard deviation were $29.4 \pm 12.2$ years). The participants were grouped in four ages 30-37 participants each (youngest, 1521, younger, 22-24, intermediate, 25-39, and older, 40-67 years). They arrived in the research unit between 8:00 and 8:30 to leave it at 9:30 next day after completing 9 sessions of resting EEG recordings separated by 3 -hr intervals (one min with eyes open and one min with eyes closed).

The experimental sleep deprivation studies were performed in accordance with the ethical standards laid down in the Declaration of Helsinki. Their protocols were approved by the Ethics Committee of the Institute of Molecular Biology and Biophysics. Informed written consent was obtained from each participant studied as paid volunteer.

\section{Assessments of Sleepiness}

Frontal and occipital electrodes were used for all EEG recordings in accord with the International 10-20 system of electrode placement. The EEG signals were recorded via a 16channel electroencephalograph (Neuron-Spectrum-2, Neurosoft, Ivanovo, Russia), conditioned by the high-pass, low-pass and notch filters $(0.5 \mathrm{~Hz}, 35 \mathrm{~Hz}$ and $50 \mathrm{~Hz}$, respectively), sampled and stored on a hard disc with a frequency of $200 \mathrm{~Hz}$.

The EEG records were visually inspected on 2-s epochs for removing from further analysis all epochs containing artifacts. The FFTW (Fastest Fourier Transform in the West) package was applied to compute power spectra for the artifactfree epochs [4]. Rectangular window taper was used on 1-s epochs without overlap for calculating absolute spectral power densities $\left(\mu \mathrm{V}^{2}\right)$ for each of 16 first single-Hz frequency bandwidth. These single-Hz power densities were averaged on each of one-min intervals of EEG record and ln-transformed. These powers were further averaged over derivations, min with eyes open and closed, and, additionally, over $4-\mathrm{Hz}$ frequency ranges. Each value for 13-25 sessions of each of 48 participants of the two-day deprivation study was subtracted from the first (evening) value (at 19:00). In additional dataset, 
single-Hz powers were similarly averaged (over derivations and $4-\mathrm{Hz}$ frequency ranges). Each value for the last 5 sessions of each of 130 participants (from 21:00 to 9:00) was subtracted from an evening value (21:00). Similarly, KSS score self-reported during each EEG recording session by using the 9-step Karolinska Sleepiness Scale [5] (a measure of subjective sleepiness) was related to a score self-reported at either 19:00 or $21: 00$ by either 48 or 130 participants of either two-day or one-night sleep deprivation study, respectively.

\section{Statistical Analyses}

Calculations were performed using the SPSS $_{23.0}$ statistical software package (IBM, Armonk, NY, USA). To correlate time courses of objective and subjective sleepiness on the interval of 25 EEG recording sessions and to relate age of study participant to subjective and objective sleepiness measures (Table I), Pearson's coefficient of correlation $(r)$ was applied.

TABLE I. AlPha Power as a CORRELATE OF AgE

\begin{tabular}{|c|c|c|c|c|c|c|c|}
\hline \multirow{2}{*}{$\begin{array}{c}\text { Clock } \\
\text { hour }\end{array}$} & \multicolumn{2}{|c|}{ Day 1 } & \multicolumn{2}{|c|}{ Day 2 } & \multirow{2}{*}{ Clock } & \multicolumn{2}{|c|}{ Night 1 } \\
\cline { 5 - 8 } & $\boldsymbol{n}$ & $\boldsymbol{r}$ & $\boldsymbol{n}$ & $\boldsymbol{r}$ & hour & $\boldsymbol{n}$ & $\boldsymbol{r}$ \\
\hline 21 & 48 & $.365^{*}$ & 42 & $.489^{* * *}$ & - & - & - \\
\hline 23 & 48 & $.280^{\wedge}$ & 42 & $.564^{* * *}$ & - & - & - \\
\hline 1 & 48 & $.338^{*}$ & 41 & $.419^{* *}$ & - & - & - \\
\hline 3 & 48 & $.400^{* *}$ & 39 & $.428^{* *}$ & 24 & 130 & .028 \\
\hline 5 & 48 & $.505^{* * *}$ & 37 & $.347^{*}$ & 3 & 130 & $.204^{*}$ \\
\hline 7 & 48 & $.387^{* *}$ & 36 & $.493^{* *}$ & 6 & 130 & $.245^{* *}$ \\
\hline 9 & 48 & $.326^{*}$ & 33 & $.361^{*}$ & 9 & 130 & $.316^{* * *}$ \\
\hline 11 & 48 & $.360^{*}$ & 29 & $.500^{* *}$ & - & - & - \\
\hline 13 & 48 & $.380^{* *}$ & 27 & $.490^{* *}$ & - & - & - \\
\hline 15 & 48 & $.321^{*}$ & 27 & $.390^{*}$ & - & - & - \\
\hline 17 & 48 & $.312^{*}$ & 25 & .217 & - & - & - \\
\hline 19 & 48 & $.438^{* *}$ & 25 & $.478^{*}$ & - & - & - \\
\hline
\end{tabular}

Notes. Objective sleepiness measure was alpha power related to either 19:00 or 21:00 in either twoday or one-night sleep deprivation study; n: Number of study participants (it is gradually decreasing throughout Day 2 due to dropouts); $r$ : Pearson coefficient of correlation between age of study participant and alpha power; ${ }^{\mathrm{p}}<0.1, " \mathrm{p}<0.05,{ }^{* * *} \mathrm{p}<0.01,{ }^{* * * *} \mathrm{p}<0.001$.

Moreover, two-way repeated measure ANOVA (rANOVA) was run to test significance of main effect of independent factor "Age" (Table II). The repeated measure was clock hour of the day 1 and day 2 (between 21:00 and 19:00) and for the night 1 (from 24:00 to 9:00).

TABLE II. MAIN EFFeCt OF INDEPENDENT FACTOR “AgE"

\begin{tabular}{|c|c|c|c|c|c|c|}
\hline \multirow{2}{*}{$\begin{array}{c}\text { Sleepiness } \\
\text { index }\end{array}$} & \multicolumn{2}{|c|}{ Day 1 } & \multicolumn{2}{c|}{ Day 2 } & \multicolumn{2}{c|}{ Night 1 } \\
\cline { 2 - 7 } & $\boldsymbol{d} \boldsymbol{f}$ & $\boldsymbol{F}$ & $\boldsymbol{d} \boldsymbol{f}$ & $\boldsymbol{F}$ & $\boldsymbol{d} \boldsymbol{f}$ & $\boldsymbol{F}$ \\
\hline Alpha power & $2 / 45$ & $5.319^{* *}$ & $2 / 22$ & $4.960^{*}$ & $3 / 126$ & $3.145^{*}$ \\
\hline KSS score & $2 / 45$ & 0.479 & $2 / 22$ & 0.639 & $3 / 126$ & 0.769 \\
\hline
\end{tabular}

Notes. Objective and subjective sleepiness measures were Alpha power and KSS score related to either 19:00 or 21:00 in either two-day or one-night sleep deprivation study; df: Degree of freedom (it is smaller on Day 2 than on Day 1 due to, in total, 23 dropouts); F: F-ratio from two-way rANOVAs (the repeated measure was clock hour) for the independent factor "Age" (either three or four ages -
see Methods); $\mathrm{p}<0.05,{ }^{* *} \mathrm{p}<0.01$.

\section{RESULTS}

The strongest of the correlations between the time course of KSS score and time courses of 16 single-Hz spectral powers was found for $10 \mathrm{~Hz}$ power at the $2^{\text {nd }} \mathrm{min}$ with eyes closed. It attained a value of $<-0.980$. Similarly, such strong correlations were obtained after averaging over the first two or five min with eyes closed and over alpha frequency range $(9 \mathrm{~Hz}-12 \mathrm{~Hz})$. Therefore, the alpha power for the first two and the $1^{\text {st }}$ min within eyes closed section of the record was chosen for relating objective sleepiness with age of study participant in the two-day and one-night study, respectively. KSS score did not show a consistent pattern of correlation with age, while alpha power was found to be a significant correlate of age in almost every EEG recording session (Table I). This relationship suggesting a weaker attenuation of alpha rhythm in older participants was confirmed by two-way rANOVAs yielding significant main effect of independent factor "Age" (Table II).

\section{DISCUSSION}

Despite observable aging-related changes in the sleepwake pattern, such as going to bed early, napping and feeling sleepy during the day, etc., the experimental studies of ageassociated differences in the response of alertness and performance measures to sleep loss consistently pointed at younger rather than older people as being highly susceptible to impairment of their alertness and performance levels during extension of wakefulness beyond its normal duration. Such contradictive facts motivated us to compare younger and older people on objective and subjective sleepiness measures in sleep deprivation experiments. The results provided support for each of two hypotheses. 1. Older adults had a reduced vulnerability to sleep loss as indicated by the results of analysis of objective measures of sleepiness. 2 . In contrast, the results of analysis of the subjective sleepiness assessments failed to reveal significant difference between ages in the strength of feeling sleepy. Therefore, the answer to such a question as are younger adults become sleepier than older adults due to sleep loss depends upon whether the sleepiness was evaluated with objective or subjective method. The result supporting the second hypothesis pointed on the preserving motivational function of sleepiness in older adults despite the age-associated decline of their EEG responsiveness to sleep deprivation.

\section{ACKNOWLEDGMENT}

The experimental studies were partially funded by the Russian Foundation for Basic Research through a research grant number 19-013-00424.

\section{REFERENCES}

[1] H. P. Landolt, J. V. Rétey and M. Adam, "Reduced neurobehavioral impairment from sleep deprivation in older adults: contribution of adenosinergic mechanisms," Front Neurol., vol. 3:62, April 2012.

[2] A. A. Putilov and O. G. Donskaya, "Evidence for age-associated disinhibition of the wake drive provided by scoring principal components of the resting EEG spectrum in sleep provoking conditions," Chronobiol. Int., vol. 33, pp. 995-1008, 2016.

[3] J. Axelsson, M. Ingre, G. Kecklund, M. Lekander, K. P. Wright and T. Sundelin, "Sleepiness as motivation: a potential mechanism for how sleep deprivation affects behavior", Sleep [online, 2019 Nov 29].

[4] M. Frigo and S. G. Johnson, "The design and implementation of FFTW3”, Proc IEEE 93, pp. 216-231, 2005.

[5] T. Åkerstedt and M. Gillberg, "Subjective and objective sleepiness in the active individual," Int. J. Neurosci., Vol. 52, pp. 29-37, 1990. 